University of Wollongong

Research Online

2015

Kinky thresholds revisited: opportunity costs differ in the NE and SW quadrants

Simon Eckermann

University of Wollongong, seckerma@uow.edu.au

Follow this and additional works at: https://ro.uow.edu.au/ahsri

Research Online is the open access institutional repository for the University of Wollongong. For further information contact the UOW Library: research-pubs@uow.edu.au 


\title{
Kinky thresholds revisited: opportunity costs differ in the NE and SW quadrants
}

\begin{abstract}
Historically, a kinked threshold line on the cost-effectiveness plane at the origin was suggested due to differences in willingness to pay (WTP) for health gain with trade-offs in the north-east (NE) quadrant versus willingness to accept (WTA) cost reductions for health loss with trade-offs in the south-west (SW) quadrant. Empirically, WTA is greater than WTP for equivalent units of health, a finding supported by loss aversion under prospect theory. More recently, appropriate threshold values for health effects have been shown to require an endogenous consideration of the opportunity cost of alternative actions in budgetconstrained health systems, but also allocative and displacement inefficiency observed in health system practice. Allocative and displacement inefficiency arise in health systems where the least cost-effective program in contraction has a higher incremental cost-effectiveness ratio $($ ICER $=m$ ) than the most costeffective program in expansion $(I C E R=n)$ and displaced services $($ ICER $=d)$, respectively. The health shadow price derived by Pekarsky, $(1 n+1 d-1 m)-1$, reflects the opportunity cost of best alternative adoption and financing actions in reimbursing new technology with expected incremental costs and net effect allowing for allocative $(n<m)$, and displacement, inefficiency $(\mathrm{d}<m)$. This provides an appropriate threshold value for the NE quadrant. In this paper, I show that for trade-offs in the SW quadrant, where new strategies have lower expected net cost while lower expected net effect than current practice, the opportunity cost is contraction of the least cost-effective program, with threshold ICER $\mathrm{m}$. That is, in the SW quadrant, the cost reduction per unit of decreased effect should be compared with the appropriate opportunity cost, best alternative generation of funding. Consequently, appropriate consideration of opportunity cost produces a kink in the threshold at the origin, with the health shadow price in the NE quadrant and ICER of the least cost-effective program in contraction $(m)$ in the SW quadrant having the same general shape as that previously suggested by WTP versus WTA. The extent of this kink depends on the degree of allocative and displacement inefficiency, with no kink in the threshold line strictly only appropriate with complete allocative and displacement efficiency, that is $n=d=m$.

\section{Publication Details}

S. Eckermann, "Kinky thresholds revisited: opportunity costs differ in the NE and SW quadrants", Applied Health Economics and Health Policy 131 (2015) 7-13.
\end{abstract}


Kinky thresholds revisited: opportunity costs differ in the NE and SW quadrants

\author{
Short running title: Opportunity costs differ in NE and SW quadrants
}

\title{
Author: Simon Eckermann
}

\begin{abstract}
Historically, a kinked threshold line on the cost-effectiveness plane at the origin was suggested due to differences in willingness to pay (WTP) for health gain with tradeoffs in the north-east (NE) quadrant versus willingness to accept (WTA) cost reductions for health loss with trade-offs in the south-west (SW) quadrant. Empirically, WTA is greater than WTP for equivalent units of health, a finding supported by loss aversion under prospect theory. More recently, appropriate threshold values for health effects have been shown to require an endogenous consideration of the opportunity cost of alternative actions in budget constrained health systems, but also allocative and displacement inefficiency observed in health system practice. Allocative and displacement inefficiency arise in health systems where the least cost-effective program in contraction has a higher incremental cost-effectiveness ratio (ICER $=m$ ) than the most cost-effective program in expansion (ICER $=n$ ) and displaced services ( ICER $=d$ ), respectively. The health shadow price derived by Pekarsky, $\left(\frac{1}{n}+\frac{1}{d}-\frac{1}{m}\right)^{-1}$, reflects the opportunity cost of best alternative adoption and financing actions in reimbursing new technology with expected incremental costs and net effect allowing for allocative $(n<m)$, and displacement, inefficiency $(d<m)$. This provides an appropriate threshold value for the NE quadrant. In this paper, I show that for trade-offs in the SW quadrant, where new strategies have lower expected net cost while lower expected net effect than current practice, the opportunity cost is contraction of the least cost-effective program, with threshold ICER $m$. That is, in the SW quadrant the cost reduction per unit of decreased effect should be compared with the appropriate opportunity cost, best alternative generation of funding. Consequently, appropriate consideration of opportunity cost produces a kink in the threshold at the origin, with the health shadow price in the NE quadrant and ICER of the least cost-effective program in contraction $(m)$ in the SW quadrant having the same general shape as that previously suggested by WTP vs WTA. The extent of this kink depends on the degree of allocative and displacement inefficiency, with no kink in the threshold line strictly only appropriate with complete allocative and displacement efficiency, that is $n=d=m$.
\end{abstract}

Key points for decision makers

1. Unless a health system is perfectly efficient, a kink in the threshold value for effects arises on the cost-effectiveness plane as opportunity costs on the south-west (SW) quadrant relate to generating funding for least loss while the north-east (NE) quadrant requires both best expansion in adopting and least loss in financing.

2. Use of the health shadow price in the NE quadrant and incremental costeffectiveness ratio (ICER) of the least cost-effective current program for 
contraction in the SE quadrant creates appropriate incentives for best expansion and contraction of current programs and technologies and associated research.

3. Given evidence of UK health system inefficiencies, current National Institute for Health and Care Excellence (NICE) decision-maker threshold values for effects are too high for opportunity costs in the $\mathrm{NE}$ quadrant and too low for opportunity costs in the SW quadrant. 


\section{Introduction - previous notion of a kinked threshold}

Under loss aversion from prospect theory [1] the values that people are willing to accept (WTA) compensation for a health loss are expected to be greater than what they are willing to pay (WTP) to achieve an equivalent health gain. O'Brien et al. [2] and Willan et al. [3], in surveying such studies in healthcare found that empirically the WTA for health loss had a value of two or more fold greater than the WTP for an equivalent health gain. Considering threshold values as determined by WTP for health gains or accept health losses relative to an origin on the cost-effectiveness plane representing the endowment of health services for patients with current practice, they consequently suggest:

(i) higher values on the cost-effectiveness plane, with losses in health relative to gains in health and;

(ii) a threshold line kinked at the origin, with greater slope (higher values) in the south-west (SW) quadrant, with negative incremental effects and costs, than on the north-east (NE) quadrant, with positive incremental cost and effects.

However, to optimise health outcomes from a fixed budget and available technology, threshold values for reimbursing new technology need to reflect budget constraints and opportunity costs of actions given existing programs and technology [4], which neither WTA or WTP allow for. The threshold value of effects to compare with when investing in new technology should be endogenously determined, reflecting opportunity cost given best alternative actions with constraints of available resources and options. 
Pekarsky [5] shows that, given a fixed budget, the opportunity cost of investing in new technology with additional net costs is the best alternative investment with adoption and financing actions. The health shadow price was subsequently derived [5] as:

$$
\left(\frac{1}{n}+\frac{1}{d}-\frac{1}{m}\right)^{-1},
$$

where: $n$ is the incremental cost-effectiveness ratio (ICER) of the most cost-effective expansion of current programs;

$m$ is the ICER of the least cost-effective current program for contraction, and;

$d$ is the ICER of services displaced.

Importantly, the health shadow price allows for allocative inefficiency and displacement inefficiency $(d<m)$, characteristic of health systems [6], reflecting best alternative adoption and financing actions for technologies with higher expected costs than currently. Allocative inefficiency is present where $n<m$ and, hence, shifting resources from the least cost-effective program in contraction (ICER $=m$ ) to the most cost-effective program in expansion (ICER $=n$ ) results in expected health gains. Displacement inefficiency is present where $d<m$ and, hence, gains could have been made contracting the least costeffective program or service to fund new technology (ICER $=m$ ), rather than the services actually displaced $($ ICER $=d$ ). Allocative and displacement inefficiency currently arise in health systems in practice given factors, including the following:

(i) Changes being restricted to the margins in reallocating funding and resources between health programs or with investment in new technology with limited resources for implementation (training, knowledge transfer, 
conveying of public health messages, administration, etc.).

(ii) Lack of institutional arrangements for assessing the most cost-effective expansion of existing programs, strategies and associated technologies.

(iii) Market failure for evidence provision to identify least cost-effective currently funded programs, strategies, and technologies $(m)$ to inform efficient program displacement in practice.

Eckermann and Pekarksy [6] highlight that many recent definitions, proposals and notions of threshold values for effects as actual or least cost-effective displaced services [7-12] against which to compare new technology, implicitly and unrealistically assume perfect allocative and displacement efficiency $(n=d=m)$. These unrealistic assumptions create a disconnect with opportunity cost and associated appropriate threshold value for effects in practice, where allocative and displacement inefficiency are present. The health shadow price fixes this problem in the presence of allocative and displacement inefficiency for a fixed budget where new technologies are expected to cost more and hence require financing. In doing so, the health shadow price reveals the extent to which threshold prices for effects are overestimated with use of actual displaced services (ICER $=d$ ) or assumed least cost-effective $($ ICER $=m$ ) displaced services on the NE quadrant. Critically, in allowing for realistic health system conditions, appropriate incentives are created with the health shadow price to undertake research into the best expansion $(\operatorname{ICER}=n)$ and contraction $(\mathrm{ICER}=m$ ) of current programs and technology in adoption and displacement decisions on the NE quadrant. This addresses biases towards new technology in research and reimbursement processes that otherwise arises in these cases [5-6]. 
Nevertheless, the opportunity cost and health shadow price identified in Pekarsky [5] assumes, like other authors proposing alternative threshold values based on displaced services [7-12] critiqued in Eckermann and Pekarsky [6], that new technology overall costs more and needs to show it is more effective than the technology it replaces. That is, notions of the threshold value with trade-offs between cost and effects have been restricted to the NE quadrant on the cost-effectiveness plane with consideration of threshold values for more effective, while more costly, new technologies or strategies, allowing for the net impact of their price and downstream costs of treatment associated with effects.

In this paper, I consider what is the opportunity cost and appropriate threshold value associated with trade-offs in the SW quadrant of the cost-effectiveness plane. That is, where a proposed technology or strategy is overall less costly while less effective. I show that, except under the highly restrictive and unrealistic conditions of perfect allocative and displacement efficiency, the appropriate threshold value in the SW quadrant is greater than the health shadow price, reflecting differences in opportunity costs. Combining this finding with the health shadow price of Pekarsky [5] in the NE quadrant, a kinked threshold line is identified and illustrated on the cost-effectiveness

plane, which is in the same direction, while for different reasons to that previously suggested [2-3].

\section{Threshold values reflecting opportunity cost in the north-east (NE) and south-west (SW) quadrants}

Where new technologies are assumed more costly and more effective than current 
practice, in the NE quadrant, then the best alternative action with a fixed budget to adoption and financing of new technology following Pekarsky [5] is as follows:

(i) Adopting the most cost-effective expansion of existing programs and technology, and

(ii) Financing this investment action from a fixed budget by displacing or contracting the least cost-effective program/s at the margin.

However, does the same opportunity cost arise with a fixed budget in the SW quadrant, where a new technology is expected to overall reduce cost while lowering effects relative to a comparator of current practice?

The best alternative action in the SW quadrant is that with the least effect reduction in raising the same funds associated with the expected cost reduction. Hence, the appropriate threshold value in the SW quadrant is contraction, or displacement of, the least cost-effective current strategy or program. That is, new technologies that overall reduce expected costs and effects relative to current practice should be compared with the best alternative way of raising funds - contraction of the least cost-effective program or technology $($ ICER $=m)$.

Consequently, a kink in the threshold value is expected given the appropriate threshold value in the NE quadrant, the health shadow price $\left(\frac{1}{n}+\frac{1}{d}-\frac{1}{m}\right)^{-1} \leq n \leq d \leq$ $m$, is less than $m$ with allocative inefficiency $(n<m)$ and/or displacement inefficiency $(d$ $<m)$ characteristic of health systems. Indeed, as highlighted in Eckermann and Pekarsky [6], with allocative inefficiency $(n<m)$ the health shadow price will be equal to $n$ if 
displacement is optimal $(d=m)$, while less than $n$ where displacement is not optimal $(d<$ $m)$, more generally.

\section{Illustrating the kinked threshold with allocative and displacement inefficiency}

To illustrate the kinked threshold, we first consider an hypothetical example for a health system where the most cost-effective expansion of current programs and technology has an ICER of \$10,000 per quality-adjusted life-year (QALY), while the least cost-effective current program or technology has an ICER of $\$ 100,000$ per QALY. If displacement were efficient, that is the least cost-effective programs or technologies were contracted or displaced in practice (i.e. $d=m=\$ 100,000 / \mathrm{QALY}$ ), then the health shadow price would be as follows: $\frac{1}{\left(\frac{1}{10,000}+\frac{1}{100,000}-\frac{1}{100,000}\right)}=10,000 / \mathrm{QALY}$ gained.

If displacement were inefficient (likely with market failure in provision of evidence for programs to displace and programs actually displaced) and actual displaced services had an ICER of say $\$ 50,000$ per QALY, then the health shadow price would be as follows:

$\frac{1}{\left(\frac{1}{10,000}+\frac{1}{50,000}-\frac{1}{100,000}\right)}=\$ 9090 /$ QALY.

This is lower than in the case of efficient displacement, as displacement, as well as adoption, can be improved in determining the highest-value alternative opportunity cost and associated threshold value. These health shadow prices reflect relevant decision contexts and conditions for opportunity costs with investments that, overall, cost more represent the threshold value that new technology should be compared against in the NE quadrant. That is, if the new technology is expected to be more effective while costing 
more than existing practice, allowing for direct and downstream costs. However, if a new technology were expected to be less costly while less effective (SW quadrant), then the opportunity cost to this investment is the best alternative raising of funds with the contraction of least cost-effective current programs and technology. Hence, the best alternative action and threshold value for the SW quadrant ICER should be that of the least cost-effective technology in contraction, \$100,000 saved per QALY lost in our example.

\section{A kinked threshold of the cost-effectiveness plane where opportunity costs differ with inefficiency}

Only under unrealistic conditions of a health system having complete allocative and displacement efficiency $(n=d=m)$ in practice does the health shadow price coincide with $m$, and only because $n=m=d$ at this single point of perfect efficiency. Hence, in reflecting opportunity cost under conditions of allocative and /or displacement inefficiency, the threshold value to compare investment in the SW quadrant will be greater than the threshold value in the NE quadrant. That is, the threshold value, $m$ in the

SE quadrant is higher than the health shadow price $\left(\frac{1}{n}+\frac{1}{d}-\frac{1}{m}\right)^{-1}$ in the NE quadrant where $n<m$ and/or $d<m$. This implies that the slope of any threshold will be greater in the SW quadrant than NE quadrant and lead to a kink, as shown in Fig. 1 for our example. 


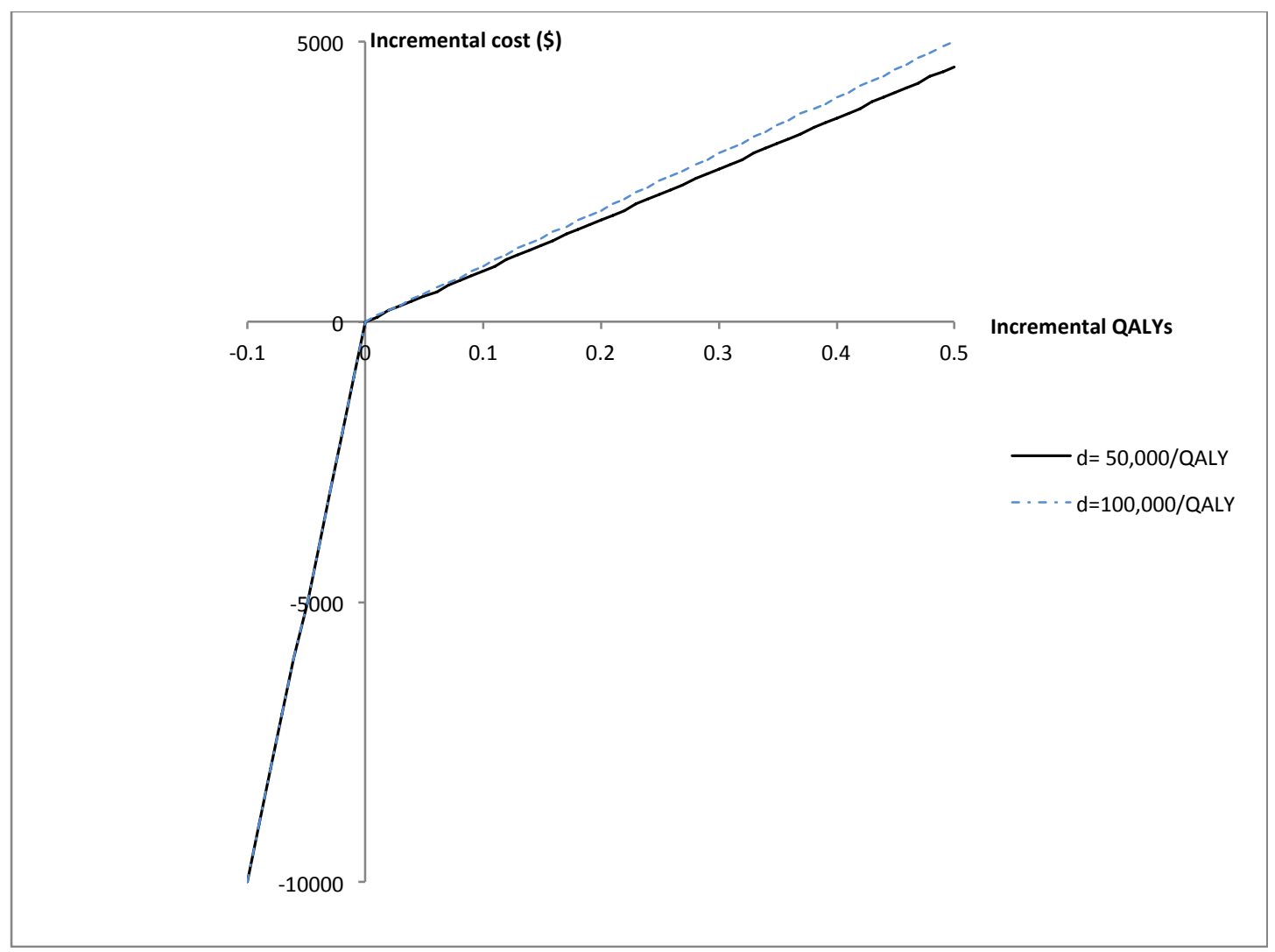

Fig1: Kinked thresholds on the cost-effectiveness plane with $n=\$ 10,000 / \mathrm{QALY}, m=$ $\$ 100,000 / \mathrm{QALY}$ and $d=\$ 50,000 / \mathrm{QALY}$ or $\$ 100,000 / \mathrm{QALY}$. QALY quality-adjusted life-year

Note that the threshold is qualitatively, as well as quantitatively, different in the SW quadrant compared with the NE quadrant. As Willan and Briggs [13] highlight, in the SW quadrant the ICER of a new technology needs to be greater than the threshold to allow cost savings greater than the value of that with best alternative contraction for the equivalent reduction in health. This contrasts with the NE quadrant, where the ICER needs to be less than the threshold to allow greater health gain for equivalent costs.

The kink arises with allocative inefficiency alone (dotted line) and even more so with the increasingly realistic assumption of allocative and displacement inefficiency (solid line). In general there will be a kink unless the health system is already at a point of perfect 
allocative and displacement efficiency $(n=m=d)$. That is, unless the most costeffective expansion of current programs or technology has the same ICER as the least cost-effective contraction, in which case threshold lines would have slope $n=m=d$ on both the NE and SW quadrants.

We now consider the case of the UK health system, where research by Claxton et al [12] estimates that in 2008 the average cost per QALY lost for services displaced services $(d)$ was $£ 12,976$ per QALY, while across 23 program budget categories the most cost-effective program identified (respiratory problems) had an ICER estimate $(n)$ of $£ 2,000$ per QALY and the least cost-effective program (maternity and neonates) reflected an ICER $(m)$ of $£ 2.73$ million per QALY. Empirically, these estimates suggest appropriate UK threshold values of $£ 1,733$ per QALY in the NE quadrant (health shadow price $\left.=1 /\left(\frac{1}{2000}+\frac{1}{12,976}-\frac{1}{2,730,000}\right)\right)$ and $£ 2.73$ million per QALY in the SW quadrant. The large difference in opportunity costs and associated appropriate threshold values in the NE and SW quadrant points to how far the UK health system is from perfect allocative and displacement efficiency. The extent of allocative and displacement inefficiency suggests that National Institute for Health and Care Excellence (NICE) guidance since 2004 for threshold values between $£ 20,000$ and $£ 30,000$ per QALY [1415] has been both too high in the NE quadrant, while too low for the SW quadrant.

\section{Moving towards allocative efficiency: the importance of appropriate thresholds}

No health system can currently claim to have perfect displacement and allocative efficiency and, hence, in general, for optimal decision making, the slope should be greater in the SW than in the NE quadrant, the extent dependent on the extent of current 
allocative and displacement inefficiency in the health system. Adopting or expanding the most cost-effective strategies, and displacing or contracting the least cost effective, ensures improvement at the margins towards allocative and displacement efficiency $[5,6$, 16]. For example, the health system in Fig. 1 could move from a position of allocative $(n<m)$ and displacement inefficiency $(d<m)$ towards allocative efficiency by identifying and using at the margins in each period the best available adoption and displacement options.

\section{In what direction and how much should the appropriate threshold kink?}

The above analysis and illustrations imply that appropriate use of opportunity costs to inform threshold values in the NE and SW quadrants point to the same direction of kink and general shape of the threshold, as that suggested, with higher WTA for a health loss than WTP for a health gain. However, it is an empirical issue for each health system depending on their allocative $(n<m)$ and displacement $(d<m)$ inefficiency as to what the slope should be in the SW quadrant $(m)$ versus the NE quadrant with their health shadow price. A health system that has high levels of allocative and displacement inefficiency will have a large kink, while this would lessen and approach a straight line for health systems if they moved towards allocative and displacement efficiency.

\section{What prevents health systems moving toward allocative and displacement efficiency}

A secondary finding across primary care trusts (PCTs) reported in Claxton et al. [12] for the UK was that, for the four largest program budget categories, ICERs were higher for PCTs expanding their budget (£14,083 per QALY) than for those contracting their budget 
( $\$ 8441$ per QALY). For those expanding their budgets, this highlights the current lack of consideration of the most cost-effective expansion of existing technology (ICER $£ 2000$ per QALY) and bias towards adoption of new patented technology inherent in UK requirements to adopt new technology when approved by NICE [17] with an operating threshold of $£ 20,000$ to $£ 30,000$ per QALY [14-15]. If PCTs were free to expand by any means, then they should be considering the most cost-effective expansion of current technology as well as displacement of the least cost-effective programs. An alternative explanation for a higher ICER in expanding trusts was suggested in Claxton et al. [12] (pages 87-88) as representing diminishing marginal returns to extent of investment. However, if this logic were consistently applied across PCTs then, effectively, we are being asked to believe that PCTs are not only assumed to currently be operating at the point of complete allocative efficiency (clearly contradicted by evidence of allocative inefficiency inherent in the same data), but have acted by their own choice to overshoot this. That is, they are now reducing health in making marginal investment and displacement decisions. While this alternative explanation denies marginal optimizing behavior across trusts and contradicts evidence across programs, a myriad of plausible factors aside from mandated provision of new technology approved by NICE with a $£ 20,000$ to $£ 30,000$ per QALY working threshold $[14,15,17]$ could also be contributing to differences between trusts' threshold values in expansion or contraction. Such plausible contributing factors include PCTs with the ability to expand, having richer sources of discretionary funding and/or less technical inefficiency with which services are provided in practice, creating scope for higher quality of care and effect values in those trusts $[18,19]$. More generally, analysis at a PCT level points to the lack of a 
pathway provided by use of displaced services as a threshold value towards allocative and displacement efficiency, without considering the most cost-effective expansion and contraction of existing programs and technology.

\section{Pathways towards allocative and displacement efficiency}

If optimal marginal decisions were made for actions within PCTs, then with diminishing marginal returns over time for choice of activities of expansion and contraction, the threshold value in the NE quadrant would be expected to rise and the threshold value in the SW quadrant expected to fall. Hence, a final hypothetical threshold price if perfect allocative and displacement efficiency were eventually achieved can be expected to be above the current health shadow price. Consequently, some might try to suggest threshold values in the NE quadrant anywhere between the current health shadow price, $\left(\frac{1}{n}+\frac{1}{d}-\frac{1}{m}\right)^{-1}$, and the ICER of displaced services. For example they might try to argue that interventions that might be acceptable in the future should be accepted now. However, such arguments fall down:

(i) Generally in denying the opportunity cost of this investment at any point in time - in the NE quadrant the best alternative adoption and displacement reflected in the health shadow price [5] and;

(ii) In not considering profit-motivated pricing up to the threshold and expected cycling through of new technologies (invested and then disinvested in or adopted and displaced) if a rule relative to currently displaced services was consistently applied [6]. 
That is, new technologies priced up to the ICER of displaced services would be next in line to be displaced if a displaced threshold were consistently applied. Such cycling through of new technology faces costs of reversal [20-22] including:

(i) Unamortised fixed costs of investment from capital, training, learning by doing and implementational costs being spread over shorter time periods than time horizon allowed for in cost effectiveness analysis;

(ii) Direct costs of reversal from public health messages being reversed.

These costs of reversal are often significant and, with pricing up to thresholds, imply that the health system could easily go backwards over time if adoption decisions were based on a threshold value of displaced services. Consequently, the coincidence of a threshold value of $d=n=m$ where there is perfect allocative and displacement efficiency does not imply that use of $d$ provides a path towards allocative efficiency.

\section{Deeper implications of costs of reversal}

The impact of costs of reversal $[6,20-22]$ associated with investment in new technology also explains the following:

(i) The ordering in which options for strategies, interventions, and programs arise does matter.

(ii) Optimisation in any period only occurs at the margins rather than across the whole system.

(iii) The threshold rule is critical in ensuring the best options are taken up earliest rather than either entrenching or cycling through new technology allowed to 
be priced such that it is less cost effective than the best alternative.

New technologies that are less cost effective than the alternative best expansion of existing technology can become entrenched with costs of reversal that are direct rather than sunk (cost of reversing public health messages and provider behavior, retraining, etc.) and hence ex post it may be better to live with, rather than cycle through, such technology. If such direct costs of reversal are high enough, decisions to invest in new technology can become irreversible, while otherwise facing reversal costs [23].

Where costs of reversal from making an investment in new technology are sunk (capital, marketing, training costs, etc.), they should not influence the decision to reverse an existing technology. However, at the point of making the decision to invest in new technology, they are still very much costs to the system where decisions are subsequently reversed and the new technology cycled through. Hence, both direct and sunk costs of reversal associated with investing in new technologies under uncertainty imply that the choice of threshold and associated rules is critical to enable moving from allocative inefficiency towards health system optimization over time.

\section{Conclusion}

The threshold value for a unit of health represented on the cost-effectiveness plane for any given health system should reflect opportunity costs of reimbursement actions in the context of allocative and displacement inefficiency for that health system. The appropriate threshold value (slope) in the SW quadrant should reflect best alternative 
contraction of the least cost-effective programs or technologies in raising of funds (ICER $=m$ ). The appropriate threshold value in the NE quadrant is the health shadow price $\left(\left(\frac{1}{n}+\frac{1}{d}-\frac{1}{m}\right)^{-1}\right)$ derived by Pekarsky [5], which reflects the best alternative adoption of the most cost-effective expansion ( $\operatorname{ICER}=n$ ) and contraction or displacement of the least cost effective $(\mathrm{ICER}=m)$ in financing. The health shadow price will be less than $m$ unless there is perfect allocative and displacement efficiency, in which case the health shadow price is $n=d=m$.

Hence, threshold values presented on the cost-effectiveness plane should only be a straight line at the origin if the health system were currently already at the singular point of complete allocative and displacement efficiency. To move towards a point of allocative and displacement efficiency, and create appropriate incentives for research and pricing, the health shadow price should be employed in making adoption decisions and setting prices for new technologies expected to be more costly while more effective. However, the threshold value for technologies expected to be less costly while less effective should be the ICER of least cost-effective strategies that could be displaced in raising funds. Alternative threshold values and associated decision and pricing rules do not provide a direct pathway toward allocative or displacement efficiency, face the very real danger of the health system going backwards allowing for impacts of practical factors (such as costs of reversal over time), and fail to create appropriate incentives for research into the best expansion and contraction of current services, technologies, and programs.

\section{References:}

[1] Kahneman D, Tversky A. Prospect theory: an analysis of decision under risk. Econometrica 
1979; 47(2): 263-291.

[2] O'Brien BJ, Gertsen K, Willan AR, Faulkner L, Is there a kink in threshold value for costeffectiveness in health care. Health Economics 2002; 11:175-180.

[3] Willan AR, O'Brien BJ, Leyva RA. Cost-effectiveness analysis when the WTA is greater than the WTP. Statistics in Medicine 2001; 20:3251-3259.

[4] Birch S. and Gafni A. Cost effectiveness/utility analyses: Do current decision rules lead us to where we want to be? Journal of Health Economics 1992; 11:279296.

[5] Pekarsky B. Trusts, constraints and the counterfactual: reframing the political economy of new drugs. PhD Thesis, University of Adelaide; 2012.

[6] Eckermann S, Pekarsky B. Can the real opportunity cost stand up: displaced services the straw man outside the room. PharmacoEconomics. 2014; 32(4): 319- 325.

http://www.springerlink.com/openurl.asp?genre=article\&id=doi:10.1007/s40273-014-0140-3.

[7] Culyer AJ, McCabe C, Briggs A, Claxton K, Buxton M, Akehurst R, Sculpher M, Brazier J. Searching for a threshold not setting one: the role of the national Institute for health and Clinical Excellence. J Health Ser Res Policy. 2007; 12(1): 56-58.

[8] McCabe C, Claxton K, Culyer AJ. The NICE cost effectiveness threshold: What it is and what that means. Pharmacoeconomics. 2008; 26(9): 733-744.

[9] Claxton K, Briggs A, Buxton M, McCabe C, Culyer A, Walker S, Sculpher M. Value based pricing for NHS drugs: an opportunity not to be missed? BMJ 2008; 336: 251254.

[10] Griffin S, Claxton K, Sculpher M. Decision Analysis for resource allocation in health care. J Health Ser Res Policy Vol. 13, Suppl 3, 2008:23-30.

[11] Sculpher M and Claxton, K. Real Economics Needs to Reflect Real Decisions. PharmacoEconomics. 2012; 30(2): 133-136.

[12] Claxton K, Martin S, Soares M, Rice N, Spackman E, Hinde S, Devlin N, Smith P, Sculpher M. Methods for the Estimation of the NICE Cost Effectiveness Threshold Final Report (2013) Working Paper 81 Centre for Health Economics, University of York.

[13] Willan A, Briggs A. 2006. Statistical analysis of cost effectiveness data. Wiley. 
[14] Devlin N, Parkin D. (2004) Does NICE have a cost effectiveness threshold and what other factors influence its decisions? A binary choice analysis. Health Economics 13(5): 437-52.

[15] National Institute for Health and Clinical Excellence. Guide to the methods of technology appraisal. NICE: London, 2008

[16] Ruta D, Mitton C, Bate A and Donaldson C. Programme budgeting and marginal analysis: bridging the divide between doctors and managers. BMJ. 2005. 330 (25): 1501-1503.

[17] Barrett, A., Riques, T., Small, M. and Smith, R. (2006). "How much will Herceptin really cost?" British Medical Journal 333: 1118 - 20.

[18] Eckermann, S., Coelli, T., Including quality attributes in efficiency measures consistent with net benefit: creating incentives for evidence based medicine in practice, Social Science \& Medicine 2013; 76: 159-168. http://dx.doi.org/10.1016/j.socscimed.2012.10.020.

[19] Eckermann S. (2009). Funding to maximise quality of care within a budget: bringing net benefit to casemix funding. Clinical Change Working Paper number 5 2009. http://clinicalchange.flinders.edu.au/publications.html

[20] Eckermann S and Willan AR. Expected Value of Information and Decision Making in HTA. Health Economics. 2007; 16: 195-209.

[21] Eckermann S and Willan AR. The option value of delay in health technology assessment. Medical Decision Making. 2008; 28: 300-305.

[22] Eckermann S, Willan A. Optimal global VOI trials: better aligning manufacturer and decision maker interest and enabling feasible risk sharing. PharmacoEconomics. 2013: 31; 393-401.

[23] Tirole J. 1988. The Theory of Industrial Organization, MIT Press.

Acknowledgement: No funding was received in support of this manuscript and I have no conflicts of interest. I would like to thank blinded reviewers for their constructive reviews and Dr Lynnaire Sheridan for meticulous proof reading. Any errors or omissions are naturally an author responsibility. 